\title{
Diffraction and an Infrared Finite Gluon Propagator
}

\author{
E. G. S. Luna ${ }^{1,2}$ \\ ${ }^{1}$ Instituto de Física Teórica, UNESP, São Paulo State University, 01405-900, São Paulo, SP, Brazil \\ ${ }^{2}$ Instituto de Física Gleb Wataghin, Universidade Estadual de Campinas, 13083-970, Campinas, SP, Brazil
}

Received on 29 September, 2006

\begin{abstract}
We discuss some phenomenological applications of an infrared finite gluon propagator characterized by a dynamically generated gluon mass. In particular we compute the effect of the dynamical gluon mass on $p p$ and $\bar{p} p$ diffractive scattering. We also show how the data on $\gamma p$ photoproduction and hadronic $\gamma \gamma$ reactions can be derived from the $p p$ and $\bar{p} p$ forward scattering amplitudes by assuming vector meson dominance and the additive quark model.
\end{abstract}

Keywords: Diffractive dissociation; Dynamical gluon mass; Photoproduction

\section{A QCD-INSPIRED EIKONAL MODEL WITH A GLUON DYNAMICAL MASS}

Nowadays, several studies support the hypothesis that the gluon may develop a dynamical mass $[1,2]$. This dynamical gluon mass, intrinsically related to an infrared finite gluon propagator [3], and whose existence is strongly supported by recent QCD lattice simulations [4]), has been adopted in many phenomenological studies [5-7]. Hence it is natural to correlate the arbitrary mass scale that appears in QCD-inspired models with the dynamical gluon one, obtained by Cornwall [1] by means of the pinch technique in order to derive a gauge invariant Schwinger-Dyson equation for the gluon propagator. This connection can be done building a QCD-based eikonal model where the onset of the dominance of gluons in the interaction of high-energy hadrons is managed by the dynamical gluon mass scale.

A consistent calculation of high-energy hadron-hadron cross sections compatible with unitarity constraints can be automatically satisfied by use of an eikonalized treatment of the semihard parton processes. In an eikonal representation, the total cross sections is given by

$$
\sigma_{t o t}(s)=4 \pi \int_{0}^{\infty} b d b\left[1-e^{-\chi_{I}(b, s)} \cos \chi_{R}(b, s)\right],
$$

where $s$ is the square of the total center-of-mass energy and $\chi(b, s)$ is a complex eikonal function: $\chi(b, s)=\chi_{R}(b, s)+$ $i \chi_{I}(b, s)$. In terms of the proton-proton $(p p)$ and antiprotonproton $(\bar{p} p)$ scatterings, this combination reads $\chi_{p p}^{\bar{p} p}(b, s)=$ $\chi^{+}(b, s) \pm \chi^{-}(b, s)$. Following the Ref. [8], we write the even eikonal as the sum of gluon-gluon, quark-gluon, and quarkquark contributions:

$$
\begin{aligned}
\chi^{+}(b, s)= & \chi_{q q}(b, s)+\chi_{q g}(b, s)+\chi_{g g}(b, s) \\
= & i\left[\sigma_{q q}(s) W\left(b ; \mu_{q q}\right)+\sigma_{q g}(s) W\left(b ; \mu_{q g}\right)\right. \\
& \left.+\sigma_{g g}(s) W\left(b ; \mu_{g g}\right)\right] .
\end{aligned}
$$

Here $W(b ; \mu)$ is the overlap function at impact parameter space and $\sigma_{i j}(s)$ are the elementary subprocess cross sections of colliding quarks and gluons $(i, j=q, g)$. The overlap function is associated with the Fourier transform of a dipole form factor, $W(b ; \mu)=\left(\mu^{2} / 96 \pi\right)(\mu b)^{3} K_{3}(\mu b)$, where $K_{3}(x)$ is the

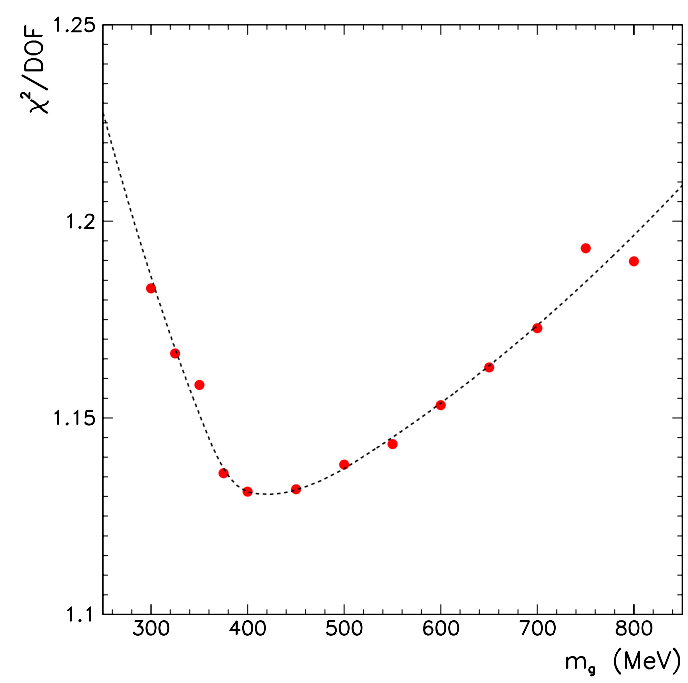

FIG. 1: The $\chi^{2} / D O F$ as a function of dynamical gluon mass $m_{g}$.

modified Bessel function of second kind. The odd eikonal $\chi^{-}(b, s)$, that accounts for the difference between $p p$ and $\bar{p} p$ channels, is parametrized as

$$
\chi^{-}(b, s)=C^{-} \Sigma \frac{m_{g}}{\sqrt{s}} e^{i \pi / 4} W\left(b ; \mu^{-}\right),
$$

where $m_{g}$ is the dynamical gluon mass and the parameters $C^{-}$and $\mu^{-}$are constants to be fitted. The factor $\Sigma$ is defined as $\Sigma \equiv 9 \pi \bar{\alpha}_{s}^{2}(0) / m_{g}^{2}$, with the dynamical coupling constant $\bar{\alpha}_{s}$ set at its frozen infrared value. The eikonal functions $\chi_{q q}(b, s)$ and $\chi_{q g}(b, s)$, needed to describe the lower-energy forward data, are simply parametrized with terms dictated by the Regge phenomenology [8]; the gluon term $\chi_{g g}(b, s)$, that dominates the asymptotic behavior of hadron-hadron total cross sections, is written as $\chi_{g g}(b, s) \equiv \sigma_{g g}(s) W\left(b ; \mu_{g g}\right)$, where

$$
\sigma_{g g}(s)=C^{\prime} \int_{4 m_{g}^{2} / s}^{1} d \tau F_{g g}(\tau) \hat{\sigma}_{g g}(\hat{s}) .
$$

Here $F_{g g}(\tau) \equiv[g \otimes g](\tau)$ is the convoluted structure function for pair $g g, C^{\prime}$ is a normalization constant and $\hat{\sigma}_{g g}(\hat{s})$ is 


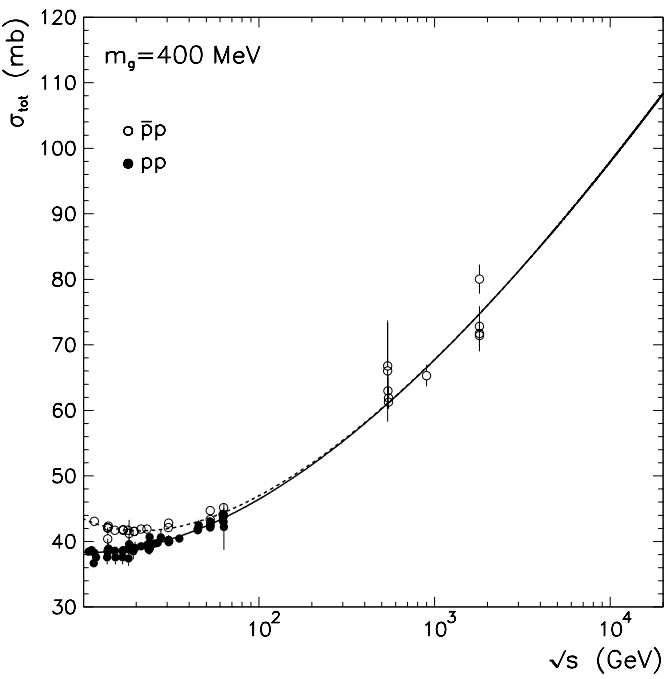

FIG. 2: Total cross section for $p p$ (solid curve) and $\bar{p} p$ (dashed curve) scattering.

the subprocess cross section, calculated using a procedure dictated by the dynamical perturbation theory [9], where amplitudes that do not vanish to all orders of perturbation theory are given by their free-field values, whereas amplitudes that vanish in all orders in perturbation theory as $\propto \exp \left(-1 / 4 \pi \alpha_{s}\right)$ are retained at lowest order. Only recently the physical meaning of the parameter $C^{\prime}$ has become fully [7]: it is a normalization factor that appears in the gluon distribution function (at small $x$ and low $Q^{2}$ ) after the resummation of soft emission in the leading $\ln (1 / x)$ approximation of $\mathrm{QCD}$,

$$
g(x)=C^{\prime} \frac{(1-x)^{5}}{x^{J}}
$$

where $J$ controls the asymptotic behavior of $\sigma_{t o t}(s)$. The results of global fits to all high-energy forward $p p$ and $\bar{p} p$ scattering data above $\sqrt{s}=10 \mathrm{GeV}$ and to the elastic differential scattering cross section for $\bar{p} p$ at $\sqrt{s}=1.8 \mathrm{TeV}$ are shown in Figs. 1 e 2 . The Figure 1 enables us to estimate a dynamical gluon mass $m_{g} \approx 400_{-100}^{+350} \mathrm{MeV}$. More details of the fit results can be seen in Ref. [8]. The results of the fits to $\sigma_{t o t}$ for both $p p$ and $\bar{p} p$ channels are displayed in Fig. 2 in the case of a dynamical gluon mass $m_{g}=400 \mathrm{MeV}$, which is the preferred value for $p p$ and $\bar{p} p$ scattering. The $\sigma_{g g}$ cross section, calculated via expression (4), is shown in Fig. 3.

\section{PHOTON-PROTON AND PHOTON-PHOTON REACTIONS}

Early modeling of hadron-hadron, photon-hadron and photon-photon cross sections within Regge theory shows a energy dependence similar to the ones of nucleon-nucleon [1012]. This universal behavior, appropriately scaled in order to take into account the differences between hadrons and the photon, can be understood as follows: at high center-of-mass

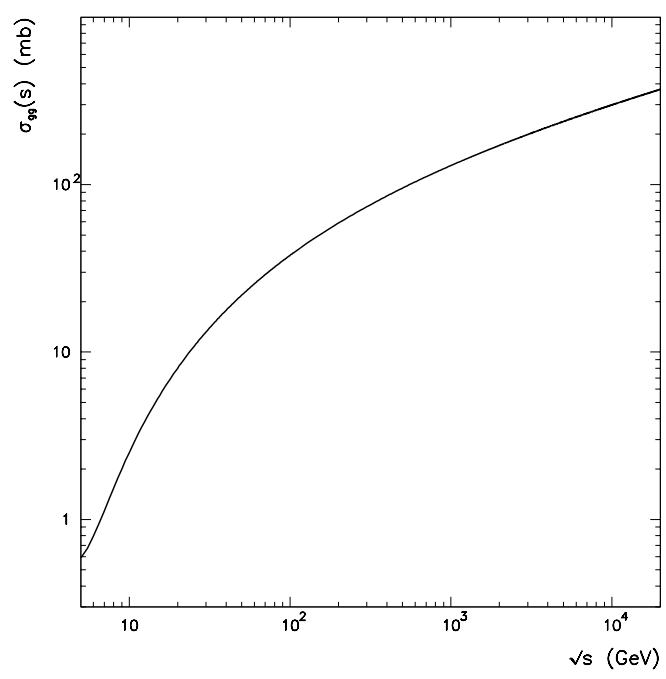

FIG. 3: Gluon-gluon total cross section. The dynamical gluon mass scale and the parameter $C^{\prime}$ were set to $m_{g}=400 \mathrm{MeV}$ and to $C^{\prime}=$ $(12.097 \pm 0.962) \times 10^{-3}$, respectively.

energies the total photoproduction $\sigma^{\gamma p}$ and the total hadronic cross section $\sigma \gamma$ for the production of hadrons in the interaction of one and two real photons, respectively, are expected to be dominated by interactions where the photon has fluctuated into a hadronic state. Therefore measuring the energy dependence of photon-induced processes should improve our understanding of the hadronic nature of the photon as well as the universal high energy behavior of total hadronic cross sections.

However the comparison of the experimental data and the theoretical prediction may present some subtleties depending on the Monte Carlo model used to analyze the data. For example, the $\gamma \gamma$ cross sections are extracted from a measurement of hadron production in $e^{+} e^{-}$processes and are strongly dependent upon the acceptance corrections to be employed. These corrections are in turn sensitive to the Monte Carlo models used in the simulation of the different components of an event, and this general procedure produces uncertainties in the determination of $\sigma^{\gamma}$ [13]. This clearly implies that any phenomenological analysis has to take properly into account the discrepancies among $\sigma^{\gamma}$ data obtained from different Monte Carlo generators. Therefore we performed global fits considering separately data of the L3 [14] and OPAL [15] collaborations obtained through the PYTHIA [16] and PHOJET [17] codes, defining two data sets as

$$
\begin{aligned}
& \text { SET I: } \sigma^{\gamma p} \text { and } \sigma_{P Y T}^{\gamma \gamma} \text { data }\left(\sqrt{s}_{\gamma p}, W_{\gamma \gamma} \geq 10 \mathrm{GeV}\right), \\
& \text { SET II: } \sigma^{\gamma p} \text { and } \sigma_{P H O}^{\gamma \gamma} \text { data }\left(\sqrt{s}_{\gamma p}, W_{\gamma} \geq 10 \mathrm{GeV}\right) \text {, }
\end{aligned}
$$

where $\sigma_{P Y T}^{\gamma \gamma}\left(\sigma_{P H O}^{\gamma \gamma}\right)$ correspond to the data of $\gamma \gamma$ total hadronic cross section obtained via the PYTHIA (PHOJET) generator.

The even and odd amplitudes for $\gamma p$ scattering can be obtained after the substitutions $\sigma_{i j} \rightarrow(2 / 3) \sigma_{i j}$ and $\mu_{i j} \rightarrow$ $\sqrt{3 / 2} \mu_{i j}$ in the eikonals (2) and (3) [13], where $\chi_{\gamma p}^{\gamma \bar{p}}=\chi_{\gamma p}^{+} \pm$ $\chi_{\gamma p}^{-}$. Assuming vector meson dominance (VMD), the $\gamma p$ total 

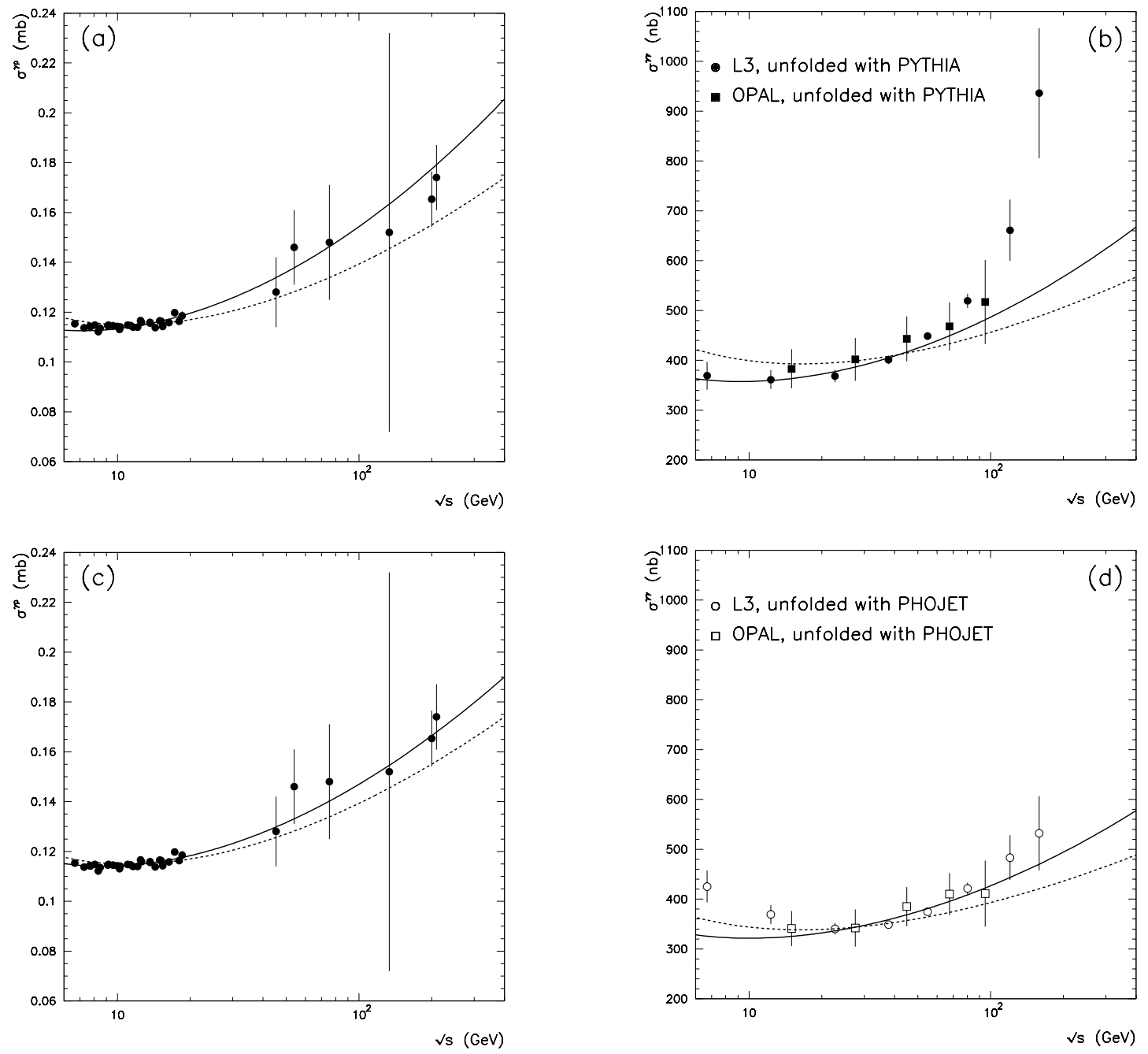

FIG. 4: $\sigma^{\gamma p}$ and $\sigma^{\gamma \gamma}$ cross sections corresponding to the case where $P_{\text {had }}^{-1}$ varies with the energy (solid curves). The dashed curves correspond to the case using a constant value of $P_{h a d}^{-1}$. The curves in (a) and (b) [(c) and (d)] are related to the SET I [SET II].

cross section is given by

$$
\sigma^{\gamma p}(s)=4 \pi P_{h a d}^{\gamma p} \int_{0}^{\infty} b d b\left[1-e^{-\chi_{I}^{\gamma p}(b, s)} \cos \chi_{R}^{\gamma p}(b, s)\right]
$$

where $P_{\text {had }}^{\gamma p}$ is the probability that the photon interacts as a hadron. In the simplest VMD formulation this probability is expected to be of $O\left(\alpha_{e m}\right)$ :

$$
P_{\text {had }}^{\gamma p}=P_{\text {had }}=\sum_{V=\rho, \omega, \phi} \frac{4 \pi \alpha_{e m}}{f_{V}^{2}} \sim \frac{1}{249}
$$

where $\rho, \omega$ and $\phi$ are vector mesons. However, there are expected contributions to $P_{\text {had }}$ other than $\rho, \omega, \phi$, as for example, of heavier vector mesons and continuum states. Moreover, the probability $P_{\text {had }}$ may also depend on the energy, which is a possibility that we explore in this work.

To extend the model to the $\gamma \gamma$ channel we just perform the substitutions $\sigma_{i j} \rightarrow(4 / 9) \sigma_{i j}$ and $\mu_{i j} \rightarrow(3 / 2) \mu_{i j}$ in the even part of the eikonal (2). The calculation leads to the following eikonalized total $\gamma \gamma$ hadronic cross section

$$
\sigma^{\gamma}(s)=4 \pi N P_{\text {had }}^{\gamma} \int_{0}^{\infty} b d b\left[1-e^{-\chi_{I}^{\gamma /}(b, s)} \cos \chi_{R}^{\gamma}(b, s)\right] \text {, }
$$

where $P_{\text {had }}^{\gamma \gamma}=P_{\text {had }}^{2}$ and $N$ is a normalization factor which takes into account the uncertainty in the extrapolation to real photons $\left(Q_{1}=Q_{2}=0\right)$ of the hadronic cross section $\sigma_{\gamma \gamma}\left(W_{\gamma \gamma}, Q_{1}^{2}, Q_{2}^{2}\right)$ [13]. With the eikonal parameters of the QCD eikonal model fixed by the $p p$ and $\bar{p} p$ data, we have performed all calculations of photoproduction and photon-photon scattering [13]. We have assumed a phenomenological expression for $P_{\text {had }}$, implying that it increases logarithmically with the square of the center of mass energy: $P_{\text {had }}=a+b \ln (s)$. The total cross section curves are depicted in Figure 3, where Figs. 3(a) and 3(b) [3(c) and 3(d)] are related to the SET I [SET II]. The results depicted in the Figures 3(c) and 3(d) show that the shape and normalization of the curves are in 
good agreement with the data deconvoluted with PHOJET [13]. The calculations using a constant value of $P_{\text {had }}$ (that does not depend on the energy $s$ ) are represented by the dashed curves. These global results indicate that a energy dependence of $P_{\text {had }}$ is favored by the photoproduction and photon-photon scattering data.

\section{CONCLUSIONS}

In this work we have investigated the influence of an infrared dynamical gluon mass scale in the calculation of $p p$, $\bar{p} p, \gamma p$ and $\gamma \gamma$ total cross sections through a QCD-inspired eikonal model. By means of the dynamical perturbation theory (DPT) we have computed the tree level $g g \rightarrow g g$ cross section taking into account the dynamical gluon mass. The connection between the subprocess cross section $\hat{\sigma}_{g g}(\hat{s})$ and these total cross sections is made via a QCD-inspired eikonal model where the onset of the dominance of gluons in the interaction of high energy hadrons is managed by the dynamical gluon mass scale. By means of a global fit to the forward $p p$ and $\bar{p} p$ scattering data and to $d \sigma^{\bar{p} p} / d t$ data at $\sqrt{s}=1.8 \mathrm{TeV}$, we have determined the best phenomenological value of the dynamical gluon mass, namely $m_{g} \approx 400_{-100}^{+350} \mathrm{MeV}$. Interestingly enough, this value is of the same order of magnitude as the value $m_{g} \approx 500 \pm 200 \mathrm{MeV}$, obtained in other calculations of strongly interacting processes. This result corroborates theoretical analysis taking into account the possibility of dynam- ical mass generation and show that, in principle, a dynamical nonperturbative gluon propagator may be used in calculations as if it were a usual (derived from Feynman rules) gluon propagator.

With the help of vector meson dominance and the additive quark model, the QCD model can successfully describe the data of the total photoproduction $\gamma p$ and total hadronic $\gamma \gamma$ cross sections. We have assumed that $P_{\text {had }}$ has a logarithmic increase with $s$. This choice leads to a improvement of the global fits, i. e. the logarithmic increase of $P_{h a d}$ with $s$ is quite favored by the data. Notice that the data of $\sigma_{P Y T}^{\gamma \gamma}$ above $\sqrt{s} \sim 100 \mathrm{GeV}$ can hardly be described by the QCD model. Assuming the correctness of the model we could say that the PHOJET generator is more appropriate to obtain the $\sigma^{\gamma \gamma}$ data above $\sqrt{s} \sim 100 \mathrm{GeV}$. This conclusion is supported by the recent result that the factorization relation does not depend on the assumption of an additive quark model, but more on the opacity of the eikonal being independent of the nature of the reaction [18].

Acknowledgments: I am pleased to dedicate this paper to Prof. Yogiro Hama, on the occasion of his 70th birthday. I am grateful to the editors of the Braz. J. Phys. who gave me the opportunity of contributing to the volume in his honor, and to M.J. Menon and A.A. Natale for useful comments. This research was supported by the Conselho Nacional de Desenvolvimento Científico e Tecnológico-CNPq under contract 151360/2004-9.
[1] J.M. Cornwall, Phys. Rev. D 26, 1453 (1982); J.M. Cornwall and J. Papavassiliou, Phys. Rev. D 40, 3474 (1989); J. Papavassiliou and J.M. Cornwall D 44, 1285 (1991).

[2] R. Alkofer and L. von Smekal, Phys. Rept. 353, 281 (2001).

[3] A.C. Aguilar, A.A. Natale, and P.S. Rodrigues da Silva, Phys. Rev. Lett. 90, 152001 (2003).

[4] F.D.R. Bonnet et al., Phys. Rev. D 64, 034501 (2001); A. Cucchieri, T. Mendes, and A. Taurines, Phys. Rev. D 67, 091502 (2003); P.O. Bowman et al., Phys. Rev. D 70, 034509 (2004); A. Sternbeck, E.-M. Ilgenfritz, M. Muller-Preussker, and A. Schiller, Phys. Rev. D 72, 014507 (2005); 73, 014502 (2006); A. Cucchieri and T. Mendes, Phys. Rev. D 73, 071502 (2006); Ph. Boucaud et al., JHEP 0606, 001 (2006).

[5] M.B. Gay Ducati, F. Halzen, and A.A. Natale, Phys. Rev. D 48, 2324 (1993); F. Halzen, G. Krein, and A.A. Natale, Phys. Rev. D 47, 295 (1993).

[6] A. Mihara and A.A. Natale, Phys. Lett. B 482, 378 (2000); A.C. Aguilar, A. Mihara, and A.A. Natale, Int. J. Mod. Phys. A 19, 249 (2004); E.G.S. Luna, Phys. Lett. B 641, 171 (2006); F. Carvalho, A.A. Natale, and C.M. Zanetti, Mod. Phys. Lett. A
21, 3021 (2006).

[7] E.G.S. Luna, A.A. Natale, and C.M. Zanetti, hep-ph/0605338.

[8] E.G.S. Luna et al., Phys. Rev. D 72, 034019 (2005).

[9] H. Pagels and S. Stokar, Phys. Rev. D 20, 2947 (1979).

[10] A. Donnachie and P.V. Landshoff, Phys. Lett. B 296, 227 (1992).

[11] R.F. Ávila, E.G.S. Luna, and M.J. Menon, Phys. Rev. D 67, 054020 (2003); Braz. J. Phys. 31, 567 (2001); E.G.S. Luna and M.J. Menon, Phys. Lett. B 565, 123 (2003).

[12] E.G.S. Luna, M.J. Menon, and J. Montanha, Nucl. Phys. A 745, 104 (2004); Braz. J. Phys. 34, 268 (2004).

[13] E.G.S. Luna and A.A. Natale, Phys. Rev. D 73, 074019 (2006).

[14] M. Acciarri et al., Phys. Lett. B 519, 33 (2001).

[15] G. Abbiendi et al., Eur. Phys. J. C 14, 199 (2000).

[16] T. Sjöstrand, Comput. Phys. Commun. 82, 74 (1994).

[17] R. Engel, Z. Phys. C 66, 203 (1995); R. Engel and J. Ranft, Phys. Rev. D 54, 4246 (1996).

[18] M.M. Block and A.B. Kaidalov, Phys. Rev. D 64, 076002 (2001). 